\title{
Observations on Aeromonas Infection in 7 Patients with Acute Leukemia
}

\author{
Huang $\mathrm{H}^{1}$, Zhao $\mathrm{J}^{1}$, Lin $\mathrm{Q}^{1}, \mathrm{Xv} \mathrm{Q}^{2}$, Fang $\mathrm{B}^{\star 1}$ and Song $\mathrm{Y}^{1}$ \\ ${ }^{1}$ Department of Hematology, Affiliated Cancer Hospital of Zhengzhou University, Zhengzhou, China \\ ${ }^{2}$ Department of Clinical Laboratory, Affiliated Cancer Hospital of Zhengzhou University, Zhengzhou, China
}

${ }^{*}$ Corresponding author: Fang B, Department of Hematology, Henan Institute of Haematology, Zhengzhou University, 127 Dongming Road, Zhengzhou, China, Fax: +86-371-65588134, Tel: +86-371-65587357, E-mail: fdation@126.com

Citation: Huang H, Zhao J, Lin Q, Xv Q, Fang B, et al. (2016) Observations on Aeromonas Infection in 7 Patients with Acute Leukemia. J Cancer Sci Clin Oncol 3(1): 101. doi: 10.15744/2394-6520.3.101

Received Date: January 04, 2016 Accepted Date: June 06, 2016 Published Date: June 08, 2016

\begin{abstract}
Backgroud: Aeromonas infections in humans are becoming increasingly frequent. They have the potential to infect humans and are associated with a variety of illnesses, such as enterocolitis, septicemia, skin and soft tissue infectious and peritonitis. However, Aeromonas is not well known, and few studies showed a comprehensive research on Aeromonas infection of different clinical features in patients with acute leukemia.

Objectives: Our study was conducted to summarize the clinical characteristics of Aeromonas infection in 7 patients with acute leukemia.

Methods: We retrospectively reviewed 7 Aeromonas infection cases occurred in our hematology ward from 2012 to 2014, analyzing the epidemiology, clinical manifestations, the pathogenic microbiology and treatment outcomes of them.

Results: In the past three years, 7 patients with acute leukemia in our department were infected by Aeromonas. There were 5 acute myelogenous leukemia patients, 2 acute lymphocytic leukemia patients. Among them, 3 patients presented Aeromonas septicemia, 1 patient presented respiratory tract infection, 1 patient presented genitourinary tract infection, and 2 patients presented necrotizing fasciitis. In our report, 3 patients (42.9\%) were dead, 2 of them died from necrotizing fasciitis, the other patient died from primary disease progression and perennial neutropenia.

Conclusion: Aeromonas infection has diversified manifestations. In addition, it can be associated with high fatality, especially cellulitis. But abundant and effective antibiotics, especially carbapenems, can significantly improve its prognosis.
\end{abstract}

Keywords: Aeromonas species; Acute Leukemia; Infection

\section{Introduction}

Reports from around the world of Aeromonas infections in humans are becoming increasingly frequent. It appears of greater clinical importance than we used to suspected [1-3]. Aeromonas species, Gram-negative, rod-shaped bacteria, are ubiquitous in the aquatic environment, located in tropical or subtropical climates. As a known etiology of diseases, Aeromonas species can cause various human illnesses, including intestinal and extra-intestinal diseases or syndromes, infectious to both immunocompetent and immunocompromised hosts systematically or locally $[4,5]$. The immunocompromised patients with hepatobiliary diseases or hematologic malignancy are most vulnerable to Aeromonas infections [6]. Among the gram-negative bacilli isolated from blood cultures of patients with acute leukemia, Aeromonas was ranked as the second frequent incidence behind Pseudomonas aeruginosa [7]. However, unlike P.aeruginosa, Aeromonas is not well known. In additon, due to the lack of a systemic report on Aeromonas infection, we could not fully master the knowledge of clinical manifestations and treatments of it. Here we retrospectively reviewed 7 Aeromonas infection cases that occurred in our hematology ward from 2012 to 2014. By analyzing the epidemiology, clinical manifestations, the pathogenic microbiology and treatment outcomes of them, we enhanced our current understanding of the rare gram-negative bacilli infections in patients with acute leukemia.

\section{Methods and features of cases}

This study was conducted at the Affiliated Cancer Hospital of Zhengzhou University, a 1000-bed hospital located in He-Nan province, China. We reviewed the case records of all patients with acute leukemia who had Aeromonas, alone or in combination with other organisms, isolated from one or more body fluids or blood culture specimens in our hematology ward during the period from 2012-2014. The diagnosis of Aeromonas infection was based on the positive results of cultures result for at least one time or the positive evidence of clinical infection. The onset of infection was regarded as being the time when the first positive result was obtained (Table 1). 


\begin{tabular}{|c|c|c|c|c|c|c|c|c|c|}
\hline $\begin{array}{c}\text { Case } \\
\text { No. }\end{array}$ & Age $(\mathbf{y r}) \&$ Sex & $\begin{array}{c}\text { Primary } \\
\text { disease }\end{array}$ & $\begin{array}{c}\text { Initial } \\
\text { ANC }(\boldsymbol{\mu l})\end{array}$ & $\begin{array}{c}\text { Initial } \\
\mathbf{B T}\left({ }^{\circ} \mathbf{C}\right)\end{array}$ & Presentation & $\begin{array}{c}\text { Source of A. } \\
\text { hydrophila }\end{array}$ & $\begin{array}{c}\text { Other bacteria isolated } \\
\text { from lesion }\end{array}$ & $\begin{array}{c}\text { Antimicrobial } \\
\text { therapy }\end{array}$ & $\begin{array}{c}\text { Treatment } \\
\text { outcome }\end{array}$ \\
\hline 1 & $21 \mathrm{M}$ & ALL & 390 & 37.3 & Pyrexia & Blood & None & IPM+TEC & Cured \\
\hline 2 & $40 \mathrm{M}$ & AML & 630 & 39.2 & Gingivitis & Throat swab & Burkholderia cepacia & IPM+VA & Cured \\
\hline 3 & $48 \mathrm{M}$ & AML & 510 & 39.4 & Cellulitis & Secretion & None & IPM+VA+SCF & Died \\
\hline 4 & $43 \mathrm{M}$ & ALL & 710 & 39 & Pneumonia & Blood & Escherichia coli & TAP+AK & Died \\
\hline 5 & $35 \mathrm{~F}$ & AML & 2170 & 37.1 & Cystitis & Urine & Citrobacter Freundii & IPM+LEV & Cured \\
\hline 6 & $21 \mathrm{~F}$ & AML & 680 & 38.8 & Cellulitis & Secretion & None & IPM +TEC+SCF & Died \\
\hline 7 & $28 \mathrm{M}$ & AML & 62 & 40.1 & Pneumonia & Blood & Pseudomonas aeruginosa & IPM+VA & Cured \\
\hline
\end{tabular}

ALL: acute lymphocytic leukemia, AML: acute myelogenous leukemia, IPM: imipenem, TEC: Teicoplanin, VA: Vancomycin, SCF: Cefoperazone and Sulbactam, TZP: Piperacillin and Tazobactam, AK: Amikacin, LEV: Levofloxacin

Table 1: Demographic data, underlying conditions, clinical presentations, infection details, and treatment outcomes of 7 patients with Aeromones hydrophil from 2012 to 2014

\section{Results}

In the past three years, 7 patients with acute leukemia in our department were infected by Aeromonas (Table 1). Most of them are middle-aged persons. Their median age was 35 (range, 21-48), 5 patients with acute myelogenous leukemia and 1 patient with acute lymphocytic leukemia. 3 patients developed simple Aeromonas infection, 4 patients developed polymicrobial infection. Among them, 2 patients (Case 1 and 5) had chronic hepatitis. 3 patients presented Aeromonas septicemia, 1 patient had respiratory tract infection, 1 patient had genitourinary tract infection, and 2 patients had necrotizing fasciitis. None of them contacted with fish or outdoor natural waters. 5 patients were infected by Aeromonas in the summer, while the other 2 were infected in the spring.

During 10 days before the onset of infections, chemotherapy (for 7 patients) and neutropenia $(<1,000 / \mu l)$ (for 6 patients) were the most common conditions, followed by central venous catheterization (except for patient 2 and 6). Temperatures were elevated to $38.5^{\circ} \mathrm{C}$ or higher in 4 patients (57.1\%). Patient $1,3,4$ and 7 had septic shock, although it was only considered to be the direct cause of death for one patient (patient 4). As for antimicrobial therapy, 6 patients received carbapenem antibiotics as their first-line therapy. In our report, among the 3 dead patients, patient 3 and 6 died from necrotizing fasciitis. Carbapenem used to be the initial treatment of myonecrosis, however, the treatment with carbapenem alone was insufficient for them. Treatments combined with vancomycin, teicoplanin, cefoperazone and sulbactam were then implemented. The minimum inhibitory concentration (MIC) of carbapenem in each case was $\leq 4$, the MIC of cefoperazone and sulbactam was $\leq 16 / 8$, and the MICs of the other two medicines were not tested. However, those patients did not respond to the treatment and died on the fifth day since onsets of infection. Patient 4 suffered from a persistent neutropenia, which led to various kinds of bacterial infections. The infections progressively developed to central nervous system, and finally result in the death of this patient.

\section{Discussion}

Aeromonas are essentially ubiquitous in the microbial biosphere. They can be isolated from virtually every environmental niche where bacterial ecosystems exist. These include aquatic habitats, fish, foods, domestic pets, invertebrate species, birds, ticks and insects, and natural soils [6]. Most studies have found the recovery of Aeromonas from fecal specimens to increase coincidentally with the warmer months of the year [8,9]. Similarly, in our case, bacterial infections were mostly observed in summer. Aeromonas have also been isolated from stool, urine and sputum samples without ascertainable pathogenicity, suggesting that they can be a constituent of normal human flora. Most available data suggests that the majority of mesophilic isolates are acquired via contact with contaminated drinking water or through the ingestion of foods (produce, dairy, or meats) [10]. However, patients in our research did not contact with outdoor fresh water or fish. In this study, A. hydrophila was the most common Aeromonas species (in all patients except for patient 6) followed by A. caviae, which is different from previous reports: A. caviae and A. veronii were the most common Aeromonas species causing bacteremia and gastroenteritis in France; A. caviae is the most frequent pathogen causing Aeromonas bacteremia in Japan; the A. hydrophila was the most common Aeromonas species causing bacteremia, followed by A. veronii biovar sobria in Taiwan [11-13]. The percentage of monomicrobic infections (42.8\%) in our study was lower than the one in previous reports (64\% to 75.6\%). When polymicrobic infections occur, Escherichia coli, Klebsiella pneumonia and staphylococcus aureus can be identified along with Aeromonas species [6,14,15]. As Altwegg [16] found, Aeromonas species were most likely to infect patients with acute leukemia among the patients with all kinds of hematologic malignancy. Aeromonas can cause mild illnesses such as intestinal and extraintestinal diseases and syndromes. Based on the frequency, Aeromonas clinical infections can be divided into four categories: (i) gastrointestinal tract syndromes, (ii) wound and soft tissue infections, (iii) bloodborne dyscrasias, and (iv) a miscellaneous "catch-all" category which includes a myriad of less frequently encountered ailments and infectious processes [7]. 
Aeromonas septicemia occurs throughout the whole year, with a higher frequency of the incidence during the summer or warmer months $[14,15,17]$. Tsai found, among hematologic malignancies, acute myelocytic leukemia (AML) ranks first in the Aeromonas infections, followed by myelodysplastic syndromes (MDS), non-Hodgkin's lymphoma (NHL), acute lymphocytic leukemia (ALL), and various other hematologic conditions, including Aplastic Anaemia (AA), thalassemia, multiple myeloma (MM), and Waldenstrom's macroglobulinemia (WM) $[15,16,18,19]$. The most common symptoms associated with Aeromonas bacteremia include fever (74\% to $89 \%$ ), jaundice (57\%), abdominal pain (16\% to $45 \%$ ), septic shock ( $40 \%$ to $45 \%$ ), and dyspnea (12\% to $24 \%$ ) $[6,14]$. Patients 1, 4 and 7 suffered from the Aeromonas septicemia, all of them presented septic shock, 2 of them had hyperpyrexia, and patient 4 got dyspnea.

The second most common anatomic matter from which Aeromonas are recovered is the integument with deeper soft tissues underlying the epidermis. Necrotizing fasciitis or myonecrosis is the most common clinical manifestation in patients with liver disease or malignancy. For patients with serious illnesses of high mortality rates (approaching 60\% to 75\%), their favorable outcomes are dependent upon the early recognition of infectious conditions, and appropriate therapeutic intervention (debridement, irrigation, antimicrobial therapy) [20,21]. Patient 3 and 6 presented necrotizing fasciitis, clinically manifesting infested limbs pains, tension blisters, swelling, blackening of the skin, subcutaneous ecchymosis, and epidermal necrolysis. Surgical intervention plus antibiotics ( $\beta$-lactam antibiotics and Glycopeptide antibiotics) were ineffective. They died eventually.

Very occasionally A. hydrophila has been described as a causal agent of respiratory tract diseases, leading to adult respiratory distress syndrome and high mortality (40\%) [22]. Those infections result in epiglottitis, empyema, lung abscesses, or pneumonia in patients with no comorbid conditions or in individuals with traditional immunocompromised states associated with the genus. By far the most frequent respiratory complication associated with the genus Aeromonas is pneumonia. Patients often present with high fever, a productive cough (hemoptysis), vomiting, chest pain, and respiratory failure [23,24]. The most common specimen found positive for Aeromonas are bloōd cultures, followed by the others like endotracheal samples, bronchoalveolar lavage or secretions, postmortem samples, such as lung and pleural effusions. Patients 4 and 7 presented pneumonia, with high fever and shiver. Patient 2 had fever, sore gums and buccal mucosal swelling. Their cultures indicated polymicrobic infections, including Aeromonas species and other gram-negative bacillis (Escherichia coli, Pseudomonas aeruginosa, Burkholderia cepacia). Aeromonas pneumonia accompanying septicemia enhanced the risk of dying. The rapid demise of patients in this setting can be as quick as 9 hours from the initial insult to the moment of death [25].

Aeromonas species are rarely implicated in infections of the urogenital tract. To our knowledge, there were four cases reported in the English-language articles, caused by A. hydrophila [26], A. veronii biotype sobria [27], A. caviae [28], and A. popoffi [29]. Patient 5 occurred hemorrhagic cystitis in the twenty-eighth day after allogeneic stem cell transplantation, characterized by frequent urination, urgency, dysuria, positive urine occult blood, and positive polyoma virus. Urine culture indicated Aeromonas hydrophila and Citrobacter Freundii infections. According to the antimicrobial susceptibility tests, the patient was cured after treatment with antibiocts (imipenem, levofloxacin) within two weeks, though Aeromonas hydrophila was reported resistant to levofloxacin.

Although the gastrointestinal tract is the most common anatomic site from which Aeromonas were recovered by far, gastrointestinal symptoms were absent from clinical manifestations in our patients. And we didn't perform stool culture. Aeromonas species can also cause ocular disease, ranging from endophthalmitis, keratitis to corneal ulceration. In addition, Aeromonas species can also cause intra-abdominal infections, such as pancreatitis, acute cholangitis, hepatic abscesses and peritonitis.

The in vitro antibiotic resistance of Aeromonas species suggests that ceftazidime, imipenem, and aztreonam should be the first-line choices for Aeromonas infection treatment [30]. Our isolated strains were sensitive to most other $\beta$ - lactams except for penicillins (such as piperacillin), quinolones and aminoglycosides, but resistant to the third generation cephalosporin (cefotaxime), piperacillin tazobactam, tobramycin, cotrimoxazole. Six-sevenths of our patients treated with imipenem obtained satisfactory effects, even for the hemorrhagic cystitis patient (patient 5) who was treated in combination with the levofloxacin which were considered to be resistant in most reports. Therefore, the combination therapy is reckoned to be better than monotherapy. Before we analyzed the blood culture results, our empirical therapy for febrile cancer patients with neutropenia was anti-Pseudomonas $\beta$ - lactam antibiotics with or without aminoglycosides, which could kill the vast majority of clinical gas Aeromonas species.

In conclusion, we reported the clinical characteristics of Aeromonas infection in 7 patients with acute leukemia in China. Aeromonas species are not rare in clinical, it can be associated with high fatality. The overall mortality rate was $43 \%$ (3/7) in the present study, higher than 35\% (6/17) in the series of Hisashi reported in 1997 [31]. The mortality rate reached up to almost $60 \%$ before 1980 [32]. Improved prognosis in recent years is largely attributed to both the substantial improvement of hematologic malignancy treatments and the advance potent anti-Aeromonas activity of $\beta$ - lactam antibiotics, such as carbapenems and new cephalosporins.

\section{Acknowledgments}

This work was supported by the department of medical record managemant of Affiliated Cancer Hospital of Zhengzhou University. 


\section{References}

1. Huang D, Zhao Y, Jiang Y, Li Z, Yang W, et al. (2015) Spontaneous bacterial peritonitis caused by Aeromonas Caviae in a patient with cirrhosis. Zhong Nan Da Xue Xue Bao Yi Xue Ban 40: 341-4.

2. Batra P, Mathur P, Misra MC (2015) Aeromonas spp as a causative agent for nosocomial infection in trauma patients. J Infect 70: 687-9.

3. Rausch S (2014) Accidental diagnosis: Aeromonas infection leading to discovery of acute myeloid leukemia with meylodysplastic syndrome. Lab Med 45: e96100.

4. Janda JM, Abbott SL (2010) The genus Aeromonas: taxonomy, pathogenicity, and infection. Clin Microbiol Rev 23: $35-73$.

5. Parker JL, Shaw JG (2011) Aeromonas spp. clinical microbiology and disease. J Infect 62: 109-18.

6. Tsai MS, Kuo CY, Wang MC, Wu HC, Chien CC, et al. (2006) Clinical features and risk factors for mortality in Aeromonas bacteremic adults with hematologic malignancies. J Microbiol Immunol Infect 39: 150-4.

7. Funada H, Machi T, Matsuda T (1988) Bacteremia complicating acute leukemia with special reference to its incidence and changing etiological patterns. Jpn J Clin Oncol 18: 239-48.

8. Edberg SC, Browne FA, Allen MJ (2007) Issues for microbial regulation: Aeromonas as a model. Crit Rev Microbiol 33: 89-100.

9. Khardori N, Fainstein V (1988) Aeromonas and Plesiomonas as etiological agents. Annu Rev Microbiol 42: 395-419.

10. Bossi-Küpfer M, Genini A, Peduzzi R, Demarta A (2007) Tracheobronchitis caused by Aeromonas veronii biovar sobria after near-drowning. J Med Microbiol 56: $1563-4$.

11. Lamy B, Kodjo A, colBVH Study Group, Laurent F (2009) Prospective nationwide study of Aeromonas infections in France. J Clin Microbiol 47: 1234-7.

12. Kimura M, Araoka H, Yoneyama A (2013) Aeromonas caviae is the most frequent pathogen amongst cases of Aeromonas bacteremia in Japan. Scand J Infect Dis 45 : 304-9.

13. Hochedez P, Hope-Rapp E, Olive C, Nicolas M, Beaucaire G, et al. (2010) Bacteremia caused by Aeromonas species [corrected] complex in the Caribbean Islands of Martinique and Guadeloupe. Am J Trop Med Hyg 83: 1123-7.

14. Lau SM, Peng MY, Chang FY (2000) Outcomes of Aeromonas bacteremia in patients with different types of underlying disease. J Microbiol Immunol Infect 33: $241-7$.

15. Llopis F, Grau I, Tubau F, Cisnal M, Pallares R (2004) Epidemiological and clinical characteristics of bacteraemia caused by Aeromonas spp. as compared with Escherichia coli and Pseudomonas aeruginosa. Scand J Infect Dis 36: 335-41.

16. Thomsen RN, Kristianse MM (2001) Three cases of bacteraemia caused by Aeromonas veronii biovar sobria. Scand J Infect Dis 33 : 718-9.

17. Ko WC, Lee HC, Chuang YC, Liu CC, Wu JJ (2000) Clinical features and therapeutic implications of 104 episodes of monomicrobial Aeromonas bacteraemia. J Infect 40: 267-73.

18. Kindschuh M, Pickering LK, Cleary TG, Ruiz-Palacios G (1987) Clinical and biochemical significance of toxin production by Aeromonas hydrophila. J Clin Microbiol 25: 916-21.

19. Cigni A, Tomasi PA, Pais A, Cossellu S, Faedda R, et al. (2003) Fatal Aeromonas hydrophila septicemia in a 16-year-old patient with thalassemia. J Pediatr Hematol Oncol 25: 674-5.

20. Cui H, Hao S, Arous E (2007) A distinct cause of necrotizing fasciitis: Aeromonas veronii biovar sobria. Surg Infect (Larchmt) 8: 523-8.

21. Lee CC, Chi CH, Lee NY, Lee HC, Chen CL, et al. (2008) Necrotizing fasciitis in patients with liver cirrhosis: predominance of monomicrobial Gram-negative bacillary infections. Diagn Microbiol Infect Dis 62: 219-25.

22. Reines HD, Cook FV (1981) Pneumonia and bacteremia due to Aeromonas hydrophila. Chest 80: 264-7.

23. Rodríguez CN, Campos R, Pastran B, Jimenez I, Garcia A, et al. (2005) Sepsis due to extended-spectrum beta-lactamase-producing Aeromonas hydrophila in a pediatric patient with diarrhea and pneumonia. Clin Infect Dis 41: 421-2.

24. Mukhopadhyay C, Bhargava A, Ayyagari A (2003) Aeromonas hydrophila and aspiration pneumonia: a diverse presentation. Yonsei Med J 44: 1087-90.

25. Miyake M, Iga K, Izumi C, Miyagawa A, Kobashi Y, et al. (2000) Rapidly progressive pneumonia due to Aeromonas hydrophila shortly after near-drowning. Intern Med 39: 1128-30.

26. Bartolomé RM, Andreu A, Xercavins M, Elcuaz R, Salcedo S (1989) Urinary tract infection by Aeromonas hydrophila in a neonate. Infect 17: 172-3.

27. Hsueh PR, Teng LJ, Lee LN, Yang PC, Chen YC, et al. (1998) Indwelling device-related and recurrent infections due to Aeromonas species. Clin Infect Dis 26: 651-8.

28. Al-Benwan K, Abbott S, Janda JM, Huys G, Albert MJ (2007) Cystitis caused by Aeromonas caviae. J Clin Microbiol 45: $2348-50$.

29. Hua HT, Bollet C, Tercian S, Drancourt M, Raoult D (2004) Aeromonas popoffii urinary tract infection. J Clin Microbiol 42: 5427-8.

30. Ko WC, Yu KW, Liu CY, Huang CT, Leu HS, et al. (1996) Increasing antibiotic resistance in clinical isolates of Aeromonas strains in Taiwan. Antimicrob Agents Chemother 40: 1260-2.

31. Funada H, Matsuda T (1997) Aeromonas bacteremia in patients with hematologic diseases. Intern Med 36: 171-4.

32. Davis WA 2nd, Kane JG, Garagusi VF (1978) Human aeromonas infections: a review of the literature and a case report of endocarditis. Medicine (Baltimore) 57: $267-77$. 


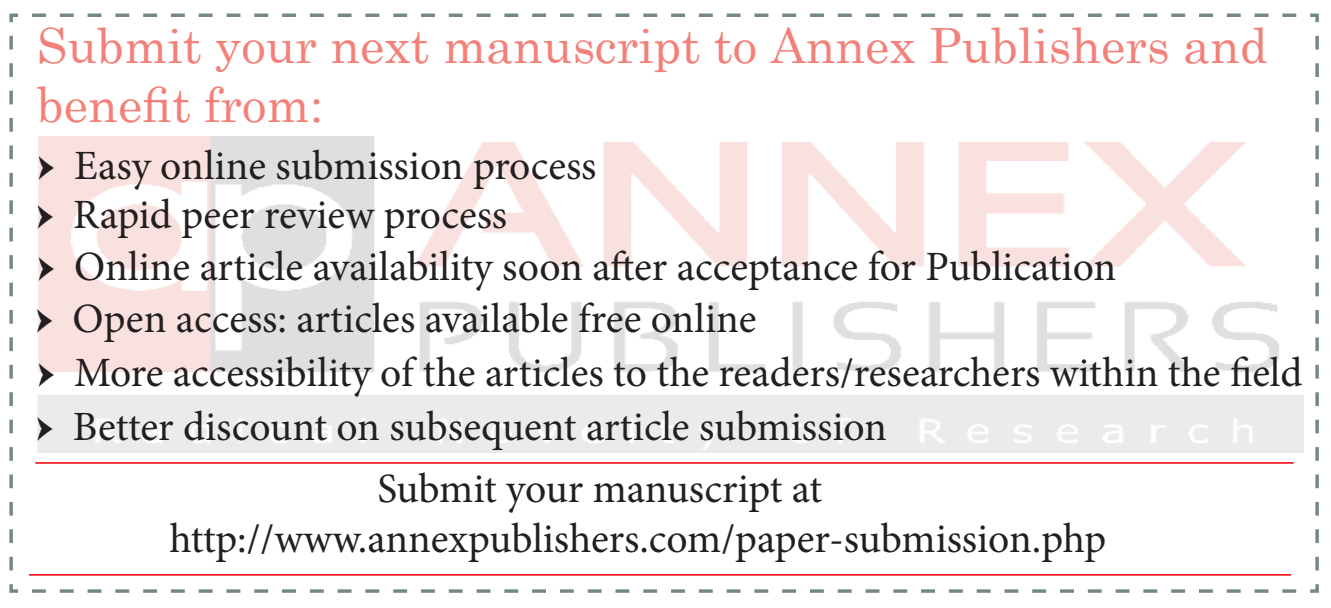

